\title{
Risks and Precautions of Genetically Modified Organisms
}

\author{
Dhan Prakash, ${ }^{1}$ Sonika Verma, ${ }^{2}$ Ranjana Bhatia, ${ }^{1}$ and B. N. Tiwary ${ }^{3}$ \\ ${ }^{1}$ Institute of Microbial Technology (CSIR), Sector 39A, Chandigarh 160036, India \\ ${ }^{2}$ Department of Biotechnology, UIET, Punjab University, Chandigarh, India \\ ${ }^{3}$ Department of Biotechnology, Guru Ghasidas Vishwavidyalaya (A Central University), Bilaspur 495009, India
}

Correspondence should be addressed to B. N. Tiwary, tiwarybn8@gmail.com

Received 9 August 2011; Accepted 18 September 2011

Academic Editor: L. Chícharo

Copyright ( $) 2011$ Dhan Prakash et al. This is an open access article distributed under the Creative Commons Attribution License, which permits unrestricted use, distribution, and reproduction in any medium, provided the original work is properly cited.

Commercial potential of biotechnology is immense since the scope of its activity covers the entire spectrum of human life. The most potent biotechnological approach is the transfer of specifically constructed gene assemblies through various techniques. However, this deliberate modification and the resulting entities thereof have become the bone of contention all over the world. Benefits aside, genetically modified organisms (GMOs) have always been considered a threat to environment and human health. In view of this, it has been considered necessary by biosafety regulations of individual countries to test the feasibility of GMOs in contained and controlled environments for any potential risks they may pose. This paper describes the various aspects of risk, its assessment, and management which are imperative in decision making regarding the safe use of GMOs. Efficient efforts are necessary for implementation of regulations. Importance of the risk assessment, management, and precautionary approach in environmental agreements and activism is also discussed.

\section{Introduction}

Modern biotechnology has allowed the movement of genetic material across unrelated species, something impossible with the traditional breeding methods. This intentional transfer of genetic material has in turn brought biotechnology out from the laboratory to the field. Genetically modified organisms (GMOs) are organisms whose genetic material has been artificially modified to change their characteristics in some way or another [1]. In essence, "genetic modification" or "genetic engineering" techniques enable scientists to find individual genes that control particular characteristics, separate them from the original source, and transfer them directly into the cells of an animal, plant, bacterium, or virus. This technology has many potential applications [2]. These new opportunities bring along greater public scrutiny and government regulation. Risk assessment is a common regulatory tool used in the decision-making process for a proposed commercial release of a GMO into the environment $[3,4]$.
Environmental applications of microorganisms are wide and varied, ranging from bioremediation, biopesticides, nitrogen fixation, plant growth promoter, to biocontrol of plant diseases, and other such agricultural practices. The sensible application of recombinant DNA techniques has shown the potential for genetically improved microorganisms to be used as soil or seed inoculants [5-8]. However, when introduced into the environment, they could have unintended environmental consequences and may play more pronounced ecological roles than the wild types [9-11]. Genetically improved microorganisms are able to reproduce and establish themselves as persistent populations and may have subtle and long-term effects on biological communities and natural ecosystems [12]. Results of DNA modification may not be limited only to the particular characteristics of the replaced gene. It is therefore important to ensure that when these organisms are released into nature they do not harm the environment or human health [13]. Such concerns have led to broader interests in the theme of risk assessment in the release of GMOs. A cautious approach 
is necessary to assess environmental risks which may occur due to introduction of recombinant organisms in the natural environment [14].

\section{Risks Related to the Use of Genetically Modified Organisms Ecological Stability of the GMO}

The application of genetic modification allows genetic material to be transferred from any species into plants or other organisms. The introduction of a gene into different cells can result in different outcomes, and the overall pattern of gene expression can be altered by the introduction of a single gene. The sequence of the gene and its role in the donor organism may have a relatively well-characterized function in the organism from which it is isolated. However, this apparent "precision" in the understanding of a gene does not mean that the consequences of the transfer are known or can be predicted [15]. Copies of a gene may be integrated, additional fragments inserted, and gene sequences rearranged and deleted - which may result in lack of operation of the genes instability or interference with other gene functions possibly cause some potential risks [16]. Therefore, there could be a number of predictable and unpredictable risks related to release of GMOs in the open environment. The report prepared by the Law Centre of IUCN, the World Conservation Union (2004), enlists numerous environmental risks likely to occur by the use of GMOs in the field. These risks are as follows.

Each gene may control several different traits in a single organism. Even the insertion of a single gene can impact the entire genome of the host resulting in unintended side effects, all of which may not be recognizable at the same time. It is difficult to predict this type of risk.

Genetic Contamination/Interbreeding. Introduced GMOs may interbreed with the wild-type or sexually compatible relatives. The novel trait may disappear in wild types unless it confers a selective advantage to the recipient. However, tolerance abilities of wild types may also develop, thus altering the native species' ecological relationship and behaviour.

Competition with Natural Species. Faster growth of GMOs can enable them to have a competitive advantage over the native organisms. This may allow them to become invasive, to spread into new habitats, and cause ecological and economic damage.

Increased Selection Pressure on Target and Nontarget Organisms. Pressure may increase on target and nontarget species to adapt to the introduced changes as if to a geological change or a natural selection pressure causing them to evolve distinct resistant populations.

Ecosystem Impacts. The effects of changes in a single species may extend well beyond to the ecosystem. Single impacts are always joined by the risk of ecosystem damage and destruction.

Impossibility of Followup. Once the GMOs have been introduced into the environment and some problems arise, it is impossible to eliminate them. Many of these risks are identical to those incurred with regards to the introduction of naturally or conventionally bred species. But still this does not suggest that GMOs are safe or beneficial, nor that they should be less scrutinized.

Horizontal Transfer of Recombinant Genes to Other Microorganisms. One risk of particular concern relating to GMOs is the risk of horizontal gene transfer (HGT). HGT is the acquisition of foreign genes (via transformation, transduction, and conjugation) by organisms in a variety of environmental situations. It occurs especially in response to changing environments and provides organisms, especially prokaryotes, with access to genes other than those that can be inherited $[17,18]$.

HGT of an introduced gene from a GMO may confer a novel trait in another organism, which could be a source of potential harm to the health of people or the environment. For example, the transfer of antibiotic resistance genes to a pathogen has the potential to compromise human or animal therapy [19]. HGT has been observed for many different bacteria, for many genes, and in many different environments. It would therefore be a mistake to suppose that recombinant genes would not spread to other bacteria, unless precautions are taken. Recent evidence from the HGT technology confirms that transgenic DNA in GM crops and products can spread by being taken up directly by viruses and bacteria as well as plant and animals cells. Very recently, Yoshida et al. [20] reported that HGT also moved from a nuclear monocot gene into the genome of the eudicot parasite witchweed, which infects many grass species in Africa.

Some of the important potential impacts of HGT from GMOs include the following [21].

Adverse Effects on the Health of People or the Environment. These include enhanced pathogenicity, emergence of a new disease, pest or weed, increased disease burden if the recipient organism is a pathogenic microorganism or virus, increased weed or pest burden if the recipient organism is a plant or invertebrate, and adverse effects on species, communities, or ecosystems.

Unpredictable and Unintended Effects. HGT may transfer the introduced genes from a GMO to potential pests or pathogens and many yet to be identified organisms. This may alter the ecological niche or ecological potential of the recipient organism [9] and even bring about unexpected changes in structure or function [22]. Furthermore, the gene transferred may insert at variable sites of the recipient gene, not only introducing a novel gene but also disrupting an endogenous gene, causing unpredictable and unintended effects. 
Loss of Management Control Measures. Regulatory approvals for field trials of GMOs often require measures to limit and control the release in space and time. With the spread of the introduced gene(s) to another species by HGT, a new GMO is created. This new GMO may give rise to adverse effects which are not controlled by management measures imposed by the original license or permit.

Long-Term Effects. Sometimes the impact of HGT may be more severe in the long term. Even under relatively strong selection pressure, it may take thousands of generations for a recipient organism to become the dominant form in the population [23]. In addition, other factors such as timing of appropriate biotic or abiotic environmental conditions and additional changes in the recipient organism could delay adverse effects.

Ethical Concerns. Various ethical issues associated with HGT from GMOs have been raised including perceived threats to the integrity and intrinsic value of the organisms involved, to the concept of natural order and integrity of species, and to the integrity of the ecosystems in which the genetically modified organism occurs [24].

Several scientific evidence that has emerged on GMOs over the last couple of years shows that there are several clear risks to human health and the environment. When genetic engineers create GMO or transgenic plants, they have no means of inserting the gene in a particular position. The gene ends up in a random location in the genetic material, and its position is not usually identified $[25,26]$. There are already several examples of such undesired effects being identified in the US after approval (e.g., GM cotton with deformed cotton bolls; increased lignin in GM soya, etc.) [27]. Releasing genetically modified plants or crop into the environment may have direct effects, including gene transfer to wild relatives or conventional crops, weediness, trait effects on nontarget species, and other unintended effects [28].

It is widely accepted that the gene flow from GM crops is possible through pollen, from open-pollinated varieties crossing with local crops or wild relatives [29]. Because gene flow has happened for millennia between land races and conventionally bred crops, it is reasonable to expect that it could also happen with transgenic crops. Transgenic crops vary in their tendency to outcross, and the ability to outcross depends on the presence of sexually compatible wild relatives or crops, which varies according to location. However, some lines of evidence suggested that whether or not gene flow between transgenic crops and wild relatives matters, in and of itself [15]. If a resulting transgenic/wild hybrid had some competitive advantage over the wild population, it could persist in the environment and potentially disrupt the ecosystem $[28,30]$.

In addition, some indirect effects of GMO were also observed which potentially harm to the environment. For example, some transgenic traits such as the pesticidal toxins expressed by Bt genes may affect nontarget species as well as the crop pests. It could happen but still uncertain how likely it is $[31,32]$. The toxicological studies of Monarch butterfly provide excellent examples, which established the sensitivity of Monarch larvae to consuming Cryl Ab protein from Bacillus thuringiensis $(\mathrm{Bt})$ expressed in transgenic maize [33], thereby triggering further to assess exposure and population level effects [31]. It was determined that larval exposure to pollen on a population-wide basis was low, given the proportion of larvae in maize fields during pollen shed, the proportion of fields planted in Bt maize, and the levels of pollen within and around maize fields that exceed the toxicity threshold $[29,30]$. However, an acute dose, even if several times higher than would be expected in the field, is not equivalent to a low natural chronic dose experienced over a longer period; therefore, a two-year study was undertaken and subsequently demonstrated that the risk to Monarch butterfly populations is $0.6 \%$ of the total of Monarch butterflies breeding in the North American Corn Belt $[31,34]$. These results indicated negligible effects of $\mathrm{Bt}$ pollen to Monarch butterfly larvae from extended exposures in field settings.

Extensive long-term use of herbicides glyphosate and gluphosinate in the Bt crops can promote the development of resistant insect pests and weeds. The Royal Society in the year 2003 has published the results of extensive farmscale evaluations of the impacts of transgenic HT maize, spring oilseed rape (canola), and sugar beet on biodiversity in the United Kingdom. These studies found that the main effect of these crops compared with conventional cropping practices was on weed vegetation, with consequent effects on the herbivores, pollinators, and other populations that are feed on it. These groups were negatively affected in the case of transgenic HT sugar beet, were, positive in case of HT Maiza and showed no effect in spring oilseed rape. However, there is still insufficient evidence to predict what the long-term impacts of transgenic HT crops will be on weed populations and associated in-crop biodiversity.

Most of the ecologists agree that gene flow is not an environmental problem unless it leads to undesirable consequences. In the short term, the spread of transgenic herbicide resistance via gene flow may create logistical and/or economic problems. Over the long term, transgenes that confirm resistance to pests and environmental stress and/or lead to greater seed production have the greatest likelihood of aiding weeds or harming nontarget species [1]. However, these outcomes seem unlikely for most currently grown transgenic crops. Many transgenic traits are likely to be innocuous from an environmental standpoint, and some could lead to more sustainable agricultural practices.

\section{Risk Assessment}

Risk is ubiquitous and unavoidable. To a great extent, therefore, our modus operandi involves assessment and management of risk. Directly observable risks are assessed and managed through heuristic processes. This direct observation may sometimes be insufficient to establish the nature and extent of risk. In such cases, we rely on other institutions, especially reputation and the rule of law [35]. Biosafety issues pertaining to the marketing of GMOs have received increasing attention by national and international agencies 
and regulatory bodies worldwide $[2,4,36]$. These are based on a common set of principles built on the accumulation of experience and scientific knowledge over the past decades.

Risk assessment intends to quantify risks and evaluate the probabilities of possible outcomes on the basis of scientific data. It is a fundamental part of improving quality, being the quality of products or the quality of life, and plays a central role in the innovation required to maximize benefits. A critical step in risk assessment is identification of circumstances that may give rise to an adverse effect(s) (risk identification or "what could go wrong" step) [25]. The level of risk is then estimated from both the likelihood ("how likely is it to happen" step) and severity/consequences ("would it be a problem" step) associated with the circumstances of concern. This is then followed by characterization of the risk based on evaluation of likelihood and consequences of the identified adverse effects being realized ("what is the risk" step) [11].

The international agreements such as Cartagena Protocol on Biosafety, Convention on Biological Diversity (CBD), and the International Plant Protection Convention (IPPC) address the environmental aspects of GMOs. The Article 15 of the Cartagena Protocol on Biosafety (CBD 2000) implies risk assessment to be in compliance with criteria of science and transparency using already existing and recognized techniques. The characterization process should adopt a multidisciplinary approach that

(i) analyses methodologies in statistics,

(ii) considers the individual components employed to produce the GMOs (such as characteristics of the donor organism, vector, and inserted DNA),

(iii) evaluates the final result in its totality (characteristics of the organism with new traits, information related to intended use, and characteristics of the potential receiving environment),

(iv) considers relevant information produced from both public and private research institutes and from international agencies.

The Cartagena Protocol on Biosafety in the year 2006 introduced an Annex III in the protocol of Article 15 for scientifically sound and transparent risk assessment taking into account risk assessment techniques. Such risk assessments shall be based at a minimum, on information provided in Article 8, and other available scientific evidence in order to identify and evaluate the possible adverse effects on human health and environment. The principles and methodology described in Annex III of the protocol follows the proven, well-accepted risk assessment paradigm, including identification of potential harmful characteristics of modified organisms that may have an adverse effect. Risk are then to be evaluated based on a combined analysis of the likelihood of the identified risks materializing and their consequences. The general principle of this protocol includes the following:

(i) lack of scientific knowledge or scientific consensus should not necessarily be interpreted as indicating a particular level of risk, an absence of risk, or an acceptable risk; (ii) risks should be considered in the context of risks posed by the nonmodified recipients or parental organisms;

(iii) risks should be assessed on a case-by-base basis.

In addition, the Cartagena Protocol on Biosafety evaluated the effectiveness of the protocol (COP-MOP) for risk assessment in the Article 35 in the year of 2008 for the safe transfer, handling, and use of living modified organisms (LMO) to protect the significant loss of biological diversity.

As in the year 2010, biodiversity target is approaching to revise the current Strategic Plan of the Convention and adopt a new biosafety strategic plan (2011-2020) to implementation of more effective risk assessment strategic plans to protect the risk of GMO/LMO according to the Bureau of COP-MOP/4.

Several pieces of information would be necessary for successful risk assessment prior to release of the GMO (1) molecular characteristics of the GMO with detailed information on genetic changes in the size and sequence, (2) details of the technology used to effect the genetic changes, (3) details of the genes and their properties that have been introduced and the possible effects of any other genetic change brought about in the organism, (4) automated karyotyping and chromosomal analysis, (5) growth characteristics of the GMO in comparison with the host organism, (6) nutrient, soil, climatic, and other requirements, (7) nature of interaction with other organisms, (8) nutritional, allergenicity and toxicity studies in case of products intended for use as food and feed, (9) gene flows from the GMO under normal ecological conditions and its impact on ecology in controlled field trials, and (10) the viability of hybrids plants, its biomass productivity, and chemical composition [37].

In order to assess whether a GMO will be safe for environment, most GMOs can reproduce, multiply, and spread in the environment after they are released. The genetic modification could give GM plants, animals, or microorganisms an advantage that would allow them to increase in numbers and spread in the environment. The environmental risks from GMOs will vary, depending on the characteristics of, and the interactions among, the organism, the trait introduced through the gene, and the environment. The novelty of GMOs, the fact that like all plants they will continue to reproduce after release, the complexity of natural environments and ecosystem processes, and the unknown evolutionary fate of inserted genes, all need to be considered in predicting environmental impacts. Despite the fact that the genes being transferred occur naturally in other species, there are unknown consequences which could be the change in the organism's/plants metabolism, growth rate, and/or response to external environmental factors. These consequences influence not only the GMO itself, but also the natural environment in which that organism is allowed to proliferate.

In 1986, a publication by the Organization for Economic Cooperation and Development (OECD), called "Recombinant DNA Safety Considerations," became the first intergovernmental document to address issues surrounding the use 
of GMOs. This document recommended that environmental risk assessments can be performed on a case-by-case basis. Since then, the case-by-case approaches of risk assessment for GMO have been widely accepted. However, the USA. has generally taken a product-based approach of risk assessment and Europe adopted a process-based risk assessment approach for GMO [38].

3.1. Environmental Risk Assessment. Environmental risk assessment (ERA) considers the impact of introducing a GM plant into a given environment. The ERA is concerned with evaluating the potential for harm to ecosystem components given that there is exposure to the GM plant. Importantly, the focus and degree of emphasis on elements of the ERA will change during the development process for the GM plant as the scope of environmental release ranges from confined field trials of limited extent through to larger-scale trials and seed increases in more environments, and to the final unconfined commercial release.

The risk of GMO toward the environment is conducted on a case-by-case basis, is comparative, and uses lines of evidence to arrive at a holistic understanding of the nature and degree of risk posed by the particular type of environmental release being analyzed [39]. In addition, a stepwise or tiered approach of data generation and analysis is used in order that the focus be directed to consequential concerns within the universe of possibilities.

Because the universe of possible concerns relevant to ERA is very large, the process of problem formulation is especially critical in order that the risk assessment be properly framed and conducted [23, 40, 41]. The universe of concerns generally need to be addressed with a few very specific questions within context to release most of the GMOs in the environments with special references to genetically modified plants. Does the genetic modification of the plant cause it to have attributes commonly associated with weeds in managed environments? Invasiveness in natural environments? Will the transgenic element in the GM plant move into native plant populations, and so what if it does? That is, will gene flow cause a native plant to become weedy or invasive (or more so)? Or will isolated populations become extinct through hybridization with the GM plant (gene swamping)? And will the GM plant adversely impact nontarget organisms that may be of special interest because they are beneficial, endangered, threatened, or charismatic? [12]. Problem formulation is a formal process whereby the risk assessor determines relevant considerations for risk assessment from this wide host of possible concerns.

The commercial development of a GM plant proceeds in a stepwise fashion, and environmental release in the first instance is in the form of field trials that are limited in number, size, and environments in which they occur. Finally, with commercialization, the GM plant is widely deployed with little concern for its confinement. Obviously, the nature of environmental impacts that need to be addressed, and therefore the data intensity and degree of scrutiny given these impacts in the ERA, will vary with the stage of development and scale of deployment being considered [42]. In view of this, the environmental risk assessment proceeds in a tiered fashion where the problem formulation considers the specific questions to be addressed and arrives at relevant data, and data synthesis needed to undertake the appropriate ERA [43]. Therefore, the ERA is dynamic with respect to the questions addressed, the data synthesized, and the comprehensiveness of the analysis conducted.

As the environmental risk assessment iterates through tiers, conservatism in conduct and interpretation of findings is balanced against uncertainties in the state of understanding. Thus, lower-tier ERA will be highly conservative to balance uncertainty, and as higher tiers of assessment are needed, increased understanding allows for more realistic (less conservative) appraisals [44].

Risk assessment also focused on the change brought about by genetic engineering allows for detailed consideration of the potential consequences of the change relative to the way the GM plant is intended to be used and the environments in which it may be found. One of the examples of safety for food use of corn engineered to be resistant to insect pests; the risk analyst at this stage can ask how specifically the change manifested by genetic transformation/expression of an active protein conferring insect resistance to the plant and may pose risks to consumers of food derived from the GM corn. In terms of potential genetically modified food safety, key considerations are how the change may result in toxicity or allergenicity.

\section{Risk Management}

Once a risk is assessed, it must be managed. The management of risk is an exclusively political action, resulting in a decision regarding whether to accept or not the risk previously estimated. It can take additional aspects (e.g., socioeconomic or ethical) into consideration and concerns methods used to reduce the scientifically identified risk. Many frameworks of risk assessment methodology separate risk assessment from risk management. Some frameworks, however, consider only certain aspects of risk management (e.g., monitoring) as separate from risk assessment but other aspects of risk management (e.g., consideration of risk mitigation options) to be part of risk assessment methodology, since a final characterization of risks must take into account the effects of any mitigation options that reduce risks. The important aspect is, of course, the iterative and interlinked relationship between risk assessment and risk management [3].

Often decisions are made with incomplete information, and this leads to uncertainty. This uncertainty needs to be handled to assess the impact it might have on a decision. Biosafety regulatory frameworks should serve as mechanisms for ensuring the safe use of biotechnology products without imposing unintended constraints to technology transfer.

The Article 16 of the Cartagena Protocol of Biosafety is purely relevant to risk management of GMO. The protocol establishes and maintains appropriate mechanisms and measures strategies to regulate, manage, and control risks identified in the provision of risk assessment.

(i) The potential to harmonize national regulatory frameworks thus ensures appropriate biosafety decision making based on scientific risk assessment. If 
properly implemented, the protocol has the potential to encourage innovation, development, technology transfer, and capacity building in relation to biotechnology, while also achieving the goals of conservation, sustainable agriculture, and equitable sharing of the technology's benefits.

(ii) To realize its potential, however, decisions concerning protocol implementation must be carefully considered and should not place undue burdens on a technology that possesses such great potential to contribute positively to sustainable agriculture and development throughout the world.

(iii) A first-things-first approach where initial efforts focus on bringing all parties to the protocol into compliance with it as quickly as possible. Developing further requirements or fine-tuning obligations at this stage only worsens the degree of noncompliance already in existence.

(iv) Therefore, capacity building should remain the primary area of focus under the Biosafety Protocol to ensure the safe adoption of this technology. In this regard, material exists to help national governments.

(v) The users and developers of agricultural biotechnology embrace their share of the duty in the protocol implementation process and will continue to campaign for fair, science-based regulations and assist with and contribute to effective capacity building.

Risk management process also forms a second focus of the economic/political component of the GMO biosafety issue. Whereas a risk/benefit analysis concludes that risks exist with regard to a GMO introduction or other activity, but are sufficiently outweighed by the benefits of that action, it will probably still be required both practically and legally to take steps to manage the risk and to ensure that damage will be minimized [45]. Elements of currently used and proposed risk management process include a variety of different kinds of activities. To a large extent, the specific protective measures imposed on the GMO user will be determined based on scientific factors linked to specific details of the GMO and the proposed use. These issues, too, turn on the ability of the decision maker to rely on unbiased scientific experts who are able to analyze each proposal or application and determine what controls are needed, and what the best available technologies and practices are [46].

The three important components was design for risk management. These components are impact assessment, public awareness/participation, and the design of regulatory systems. These concepts, all very important in this field, are critically important for GMO-related governance. It is not possible to overstate the importance of the public's contribution to effective decision making, as well as the importance of public awareness, within the context of government decisions on matters and activities affecting the environment [47].

4.1. Role of Impact Assessment Processes. Within the concept of risk management, the mechanism of impact assessment plays a crucial role. Although extending well beyond the scope and detail of many environmental impact assessment (EIA) procedures, the assessments mandated under national biosafety-related legislation, and especially under the Cartagena Protocol, provide a clear foundation on which at least some of a country's various decision making, permitting, labeling, and other processes relating to GMOs could be based. Unfortunately, although the need for risk assessment is undisputed, the particular parameters of that investigation are difficult to quantify in the biosafety area, given the fact that GMO introductions are a relatively new innovation. In this connection, it is important to note that the development of agreed risk management measures would provide a real benefit for both the GMOs proponents, the communities, and the ecosystems that would be most affected by the identified risks.

4.2. Public Awareness/Access to Information. Public access to information is an important cornerstone of public participation and is one of the tools that could help to realize the benefits and avoid the risks of modern biotechnology. This concept is well recognized in Principle 10 of the Rio Declaration, and in the recently adopted Aarhus Convention on access to information, public participation in decision making, and access to justice in environmental matters [12].

4.3. Transparency and Capacity. Simple "transparency"and "access" to relevant documents, however, may not be sufficient in the case of biosafety issues. Arguably, the concept of access to information must include, in some way, access to the tools and expertise with which to understand that information. While merely providing access to the data will be sufficient in many developed countries that are home to highly specialized and active NGOs, even here the balance of expertise weighs heavily on the side of the GMO proponents, often the companies or institutions that developed the GMOs [48].

4.4. Labelling, Standards, and Certification. Beyond the public's access to governmental documents and processes, however, there are other mechanisms by which public awareness and access to information can be encouraged, including product labeling, food safety standards, and general consumer protection laws, all of which are designed to foster awareness and communicate public preferences to the commercial proponents of GMOs in a way that will get their attention. These mechanisms can be effective if they are accurate, specific, and clearly expressed in understandable language, unbiased, and based on full disclosure of the relevant facts by the GMO proponents. In California, a major referendum requiring disclosures of toxic and carcinogenic substances in public places and consumer goods was basically invalidated by regulations that allowed those disclosures to be made in generic terms.

4.5. Confidential Information. One of the key concerns in this regard relates to the proponent's need to maintain 
some information as "confidential." While the basic realities of modern business clearly underscore the need for confidentiality, it is also true that confidentiality provisions are often used as a means of avoiding disclosures. In the face of increasing recognition that activities, including especially species introduction, in one country may have serious impacts on neighbouring countries, labelling and other access to information is increasingly addressed at international and regional levels [49].

4.6. Direct Public Participation and Awareness Mechanisms. With regard to direct public participation in biosafety-related decision making, a small number of countries, including Denmark, The Netherlands, and New Zealand, are also taking a leading role in developing mechanisms for public awareness. These countries' legislative provisions require relatively broad-based stakeholder processes addressing certain aspects of modern biotechnology, including the release of GMOs. Such processes help the governments and regulatory agencies to gauge public opinion, generate dialogue, gather useful information, and develop awareness within their populations on modern biotechnology [50].

\subsection{Design of Regulatory Systems for GMO Development and} Use. In many different fields of endeavour, technological capacity to act has moved significantly faster than has the governmental ability to oversee and regulate it. As a consequence, many concerns relating to the risk of GMOs are directed more closely to the apparent lack of societal and governmental restraints on GMO developers and users, rather than to addressing particular scientific issues. This suggests that a third key element of the risk-management process involves a reconsideration of regulatory mechanisms and systems for governmental oversight of GMO development and use [51].

4.8. Sociocultural Impacts. It is in the area of sociocultural impacts that the controversy over GMOs and biosafety takes on its most complex aspect. On one hand, food production, food security, and livelihood improvement are all critical elements of sustainable development, to which GMOs and other products of modern biotechnology are often cited as important contributions. On the other hand, the introduction of GMOs can affect humans, (as well as animals and ecosystems), particularly at the community level, in many ways beyond direct physical sustenance, not all of which are beneficial [52]. The environmental questions surrounding biotechnology need to be addressed, yet the technology as a whole offers great promise of environmental, social, and economic benefits that should not be inhibited unnecessarily. A number of concerns should be addressed through sociocultural assessment of the impact (sociocultural risks and benefits) of GMOs. These include the biodiversity impacts of extending GMO introductions into marginal areas (which are often centres of diversity not only of wild species but also of traditional agricultural species) and into protected areas and their buffer zones [53].

\section{Ways to Manage Risks}

Management and mitigation of risk allows feedback for validation of the initial assessment. Risks can vary depending on several factors including nature of the GMO, its intended use, and the environment receiving the GMO. Therefore, they should be assessed and managed on a case-by-case basis. The purpose of case-by-case practice is to treat every release as unique, since every GMO represents different genetic characteristics. Prior consent from the authorities is advisable in order to perform deliberate release, and field trials before the GMO may be commercialized [54, 55]. This is particularly important for genetically engineered microorganisms that have the potential to survive, persist, and spread in the environment to which they may gain access. As cited by the European food safety authority [13], the following points should be addressed when appropriate:

(i) the potential for survival and persistence in the receiving environment and any selective advantage that may be offered: in case of selective advantage, its nature should be identified along with any potential for negative effects;

(ii) the potential for gene transfer;

(iii) the potential for negative effects or consequences based on interactions with indigenous microorganisms;

(iv) possible effects on humans, animals, and plants;

(v) possible effects or (nonreversible) perturbations on biogeochemical processes.

These points may be assessed by a combination of laboratory studies, micro- and mesocosm experiments, and smallscale field releases to identify hazards and to quantify actual levels of exposure [56]. However, extrapolation of assessment from one context to another, that is, from laboratory research to small-scale field trials and finally to commercial scale is not recommended. Small-scale trials involve a lesser number of GMOs and may provide valuable information related to concerns like survival and persistence, competitive fitness, and some ecological implications of release. Commercial release, on the other hand, involves a higher number of GMOs to be released in different, complex ecosystems and needs to be carefully carried out over time and at different sites to reveal impact on relationships between species and ecosystem interactions [57].

Generally, potential risks by use of GMOs can be mitigated using risk-management strategies that may make some proposed activities acceptable. This can be achieved, for example, using confinement strategies and monitoring.

5.1. Contained Use of GMOs. The term "contained use" covers any activity involving GMOs in which measures are taken to limit contact between them and people or the environment. It relates to the actual process of genetic modification, and also to the use, storage, transport, and destruction of GMOs. Containment of GMOs can be physical or biological. Physical containment includes barriers 
designed to prevent organisms from escaping the laboratory and be accidentally released. This may involve the use of specially built laboratories, sterilization procedures, restriction of access, and so forth. Biological containment involves designing the organism in such a manner that they cannot grow out of the laboratory.

5.2. Monitoring the Fate of GMOs Released in the Environment. An important aspect in the process of GMO risk management is the postrelease monitoring phase initiating from the moment of environmental release. Regular inspections are necessary for effective monitoring, using a diversity of analyses over an extensive period of time. Monitoring will have two focuses: (1) possible effects of the GMO identified in the formal risk assessment procedure and (2) identification of the occurrence of adverse unforeseen effects of the GMO or its use that were not anticipated in the environmental risk assessment.

The establishment of monitoring procedures may be difficult, but such monitoring is essential not only to understand the effectiveness of the introduced organism but also to detect unexpected spread. In general, the procedures involve development or application of already existing techniques for identifying the organism in environmental samples. These procedures have been developed and are, in most cases, well accepted [58].

The scientific knowledge and experiences gained from monitoring will in turn inform the risk assessment process. Thus, the results of monitoring provide opportunities to update risk assessment continually in the light of any new knowledge.

Biosafety regulatory frameworks of GMO should serve as mechanisms for ensuring the safe use of biotechnology products without imposing unintended constraints to technology transfer. To be able to judge the sensitive balance between these aspects of GMO risk management, measuring the costs of biosafety regulation and the potential impacts on biotechnology research and development is crucial. A necessary first step to answering questions about the causes and consequences of the process of regulatory approval for new biotech crops is to understand the operation of the regulatory system and the size and structure of the costs of compliance. It seems that the compliance costs incurred by biotechnology developers are quite high, and the regulatory burden of novel biotech crops might be out of balance. Reflections on trends, challenges, and issues on risk assessment and management in a developing country context were presented. Biosafety regulatory frameworks were reviewed in relation to the development process, challenges, and trends in its formulation, especially in the context of risk assessment and management. The choice of a biosafety regime in the context of developing countries is influenced not only by the science-based approach in risk analysis but also by the social, political, and environmental governance mechanisms and experience gained in relation to practice and conventions within a particular country.

The regulatory systems designed to deal with GMO should reduce the amount of risk and create the social adaptive capacity necessary to cope with the risks associated with new technologies. There are many different ways to achieve these goals. However, the three separate methods for addressing these challenges are biosafety protocols, a moratorium, and insurance.

Currently, Austria, UK, and Germany have moratoriums, while the EU has a de facto moratorium effected from June, 1999 until 2003. Such moratoriums delay the introduction of GMO that could reduce the amount of ecological degradation produced by GM. However, moratoriums offer a number of benefits. A delay could provide the opportunity to develop institutions to effectively evaluate and monitor GMO. It would also allow science to better assess the potential indirect impacts of existing GM, such as the evolution of $B t$ resistance. Furthermore, a moratorium may provide the time needed to allow a richer public debate to address how to fairly balance the risks and benefits of GMO. Given the uncertainty surrounding both the likelihood and degree of potential impacts of GMO risks, it is sensible for society to purchase insurance against these risks [59]. However, due to the unknown and variable nature of risks, private insurance is virtually impossible, which forces the public to play this role. Taxes on the use of transgenic products could function as a type of social insurance, as long as such a tax was invested in ecological conservation and restoration, to mitigate against any disruption caused by GMO.

\section{Precautionary Approach}

When there is a risk, two or more outcomes are possible, which one will occur is unknown but at least one of them is undesired. It is within this context that the precautionary approach from Principle 15 of the Rio Declaration [60] can be introduced.

Precautionary approach is premised on the notion of reducing, if not eliminating, risks to human health and the environment. It acknowledges the complexity and variability of the natural environment and embodies certain humility about scientific procedures and knowledge. It prioritizes the rights of those who stand to be affected by an activity, rather than those who stand to benefit from it [57]. It involves scrutiny of all available alternatives and an examination of justifications and benefits as well as risks and costs. In short, a precautionary approach involves the adoption of longterm, holistic, and inclusive perspectives in environmental protection $[46,61,62]$.

Precautionary approach or precautionary principle is the borderline between science and policy or science and governance, in modern parlance. It is often divided into three components: (1) the lack of scientific certainty, (2) a risk of irreversible or serious damage, and (3) an obligation for states to take measures accordingly. The precautionary principle aims to replace uncertainty to ensure safety until other measures or solutions can be implemented. Within a policy that strives to achieve sustainable development over a long period, the precautionary principle seems to be indispensable. As the precautionary principle intends to protect the environment beyond current scientific knowledge, its 
implementation may not be justified or questioned on the basis of current scientific data alone.

The virtue of precautionary principle is the avoidance of risks that are impossible to assess. Its vice is that these risks, which may not even exist, can only be avoided by refusing to improve quality, being product quality or the quality of life. It has been justified by academics and pressure groups for imposing restrictions on the use of genetic modification technology claiming that the lost species and ecosystems can never be recovered for future generations. On the other hand, the development-focused environmentalists note that future generations may not come into being to appreciate these ecosystems without effective action on development imperatives.

The precautionary calculus often overlooks the fact that even when technologies introduce new risks, very often they confer net benefits-that is, their use reduces many other serious and costly hazards. For example, the use of GMOs with enhanced pest and disease resistance has reduced the use of pesticides, reducing runoff into waterways, and the exposure of workers who manufacture, transport, and apply these chemicals. It has also permitted farmers to adopt environment-friendly, no-till farming practices. Rice varieties enhanced with provitamin A and iron could drastically improve health of hundreds of millions of the malnourished in developing countries. Such tangible environmental and health benefits are usually given little or no weight in precautionary risk calculations [63].

To gain more clarity on whether the precautionary principle could be used as an effective tool to reduce the overall risks or to merely regulate risks, we can consider the experience of the European Union and the US, respectively, with the genetically modified crops [64]. The EU has ensured low rates of adoption of GM crops within Europe, but it does not follow that this has reduced overall environmental or health risks, the very reason for the precautionary approach. But the US approach, which incorporates a moderate version of the principle (requiring governmental approval prior to their commercial cultivation), has led to rapid adoption of GM crops and brought significant environmental benefits (relative to conventional crops) such as higher yields, lower pesticide usage, and increases in biodiversity [65]. Thus, while the EU's version of the precautionary principle has effectively limited GM crops by prolonging riskier practices, it has been environmentally counterproductive.

Even though several countries have adopted well-drafted environmental and biodiversity laws, reference to "precautionary principle" is missing. For instance, Malaysia's national biodiversity policy makes explicit reference to the convention on biological diversity (1992) but refrains from using the term "precautionary principle." Similarly, other countries in the region, Vietnam, Indonesia and Lao PDR, also do not directly invoke precautionary principle in their laws.

On the other hand, in several countries (e.g., India and Pakistan), the highest judicial authority has cited "precautionary principle" in its judgments. Several African countries have made explicit reference to "precautionary principle" in their laws. Examples include the 1997 Mozambique environment legislation, the 1996 general environmental law of Cameroon, and South Africa's National Environmental Management Act.

Latin American countries have also incorporated 'precaution as guiding principle' in their national environmental laws. Examples include general and biodiversity-related environmental laws in Argentina, Peru, Costa Rica, and Ecuador.

The "precautionary principle" in Australia's environmental policy is deeply rooted, as reflected in the InterGovernmental Agreement on Environment of 1992 and the Commonwealth Environment Protection and Biodiversity Conservation Act of 1999.

This "Precautionary Principle" as mentioned above is deeply entrenched in the environmental legislations of several European countries. On the other hand, in the United States of America, precaution is rarely stated explicitly in any of its laws. However, the precautionary principles are well entrenched in several protection acts such as Endangered Species Act of 1973 and the Wild Bird Conservation Act of 1992.

A report by the European Environment Agency (2002) summarizes the European experience invoking "Precautionary Principle" for environmental management which provides useful insights on what lessons can be learned about the Precautionary Principle from several case studies. These lessons include the following: (1) respond to ignorance as well as uncertainty, (2) research and monitor for early warnings, (3) search out and address "blind spots" and gaps in scientific knowledge, (4) identify and reduce interdisciplinary obstacles to learning, (5) ensure that realworld conditions are fully accounted for and systematically scrutinize and justify the claimed "pros" and "cons", (6) evaluate alternatives, (7) promote robust diverse and adaptable solutions, and (8) use "lay" and local knowledge as well as all relevant specialist expertise.

The increasing acceptance of precautionary principles has, however, been accompanied by changes in their public profile. Many European industries decreasingly view precautionary principles as acceptable risk management approaches and increasingly view them as tools for environment and health advocates. These industries 'disenchantment is fed both by decreasing control over regulatory bodies' interpretation of "precaution" and by seeing precautionary principles endorsed by individuals with very different worldviews. These proponents advocate precaution as a response to globalization and the so-called "risk society" where risks extend over time and national boundaries, without compensating many of those affected by them [63].

Some environmental groups argue that this world requires an expanded role for NGOs, representing and interpreting precautionary principles. These groups do not dismiss science but doubt its ability to resolve issues with great uncertainties and pervasive value conflicts. However, even those who acknowledge limits to conventional science may be uncomfortable with the transfer of power where NGOs made the arbiters of ambiguity [66].

Some US agencies accepted that businesses and governments may invoke precautionary principles to protect 
their markets from outside competition. They point to "precautionary" bans that fall heavily on US products, such as GM corn and beef raised with growth hormones [67]. The Commission of the European Communities [68] has recognized these possibilities without offering a clear resolution.

In contrast, the above precautionary principle is anultimate key issue within the sustainable development framework. New approaches are needed to take into account the risk, uncertainty, and complexity involved. There is a need to identify normative preferences, and the stakeholders perspectives and extended peer communities are needed.

\section{How to Ensure the Role of Precautionary Measures}

The precautionary approach offers the public and decision makers a forceful, common-sense approach to environmental and public health problems. In order to deliver the promise implied by its name, the principle should not increase the overall risks. To ensure that a policy is truly precautionary, one should compare the risks of adopting the policy against not adopting it. It should take care of some ethical criteria to ensure that it actually reduces overall risks when outcomes are ambiguous. These include the human mortality and morbidity threat, threats from outcomes that are irreversible or persistent, the immediacy criteria wherein immediate threats must be taken care of prior to threats that could occur later, and the uncertainty criteria where threats of harm that are more certain should take precedence over less certain harms.

As formulated at the "Wingspread Conference" [69], the principle of precautionary action has 4 parts.

(1) People have a duty to take anticipatory action to prevent harm.

(2) The burden of proof of harmlessness of a new technology, process, activity, or chemical lies with the proponents, not with the general public.

(3) Before using a new technology, process, or chemical, or starting a new activity, people have an obligation to examine "a full range of alternatives" including the alternative of doing nothing.

(4) Decisions applying the precautionary principle must be "open, informed and democratic" and "must include affected parties."

In February 2, 2000, European Commission Communication (ECC) released a note on the Precautionary Principle: the "precautionary principle" applies where scientific evidence is insufficient, inconclusive, or uncertain, and preliminary scientific evaluation indicates of there are reasonable grounds for concern that the potentially dangerous effects on the environment, human, animal, or plant health EC [2000].

It is important to emphasize that although this principle operates in the context of scientific uncertainty, it is considered by its proponents to be applicable only when, on the basis of the best scientific advice available, there is good reason to believe that harmful effects might occur [44]. The precautionary principle is most often applied in the context of the impact of human actions on the environment and human health, as both involve complex systems where the consequences of actions may be unpredictable

Principle 15 of the Rio Declaration remains by far the most widely quoted version of the precautionary principle. It states that in case of "threats of serious or irreversible damage, lack of full scientific certainty shall not be used as a reason for postponing cost-effective measures to prevent environmental degradation". It also brings in the element of proportionality by stating that measures should be applied according to the capability of the states.

The Cartagena Protocol on biosafety based on the Precautionary Principle, emerged as a result of international negotiation to reduce the transboundary movement transit, handling and use of living modified organism (LMO) that may negatively impinge on the biological diversity. Three are three core elements of the Cartagena Protocol: advance information agreement (AIA), risk assessment and the precautionary principle. The idea of the for requiring AIA of LMO is that states have a right to know what is coming into their territories, and the information should be provided in time to prepare possible harm. This procedure applies only to LMOs for introduction into environment. AIA procedures are embodied in Articles 8, 9, and 10. According to these procedures, the exporting party must communicate a written request to importer prior to transfer of LMOs intended to be introduced into environment.

The risk assessment of the Article 10 is envisioned in the protocol as guideline for parties in their decisions to import LMOs. An assessment of risk will enable them to anticipate and prevent environmental harm. Risk assessment should be performed with information available to importing state in the AIA documentation. Procedures to assess risk should be conducted in a scientifically sound manner. This article also contains explicit support for the risk management and precautionary approach.

Article 11 of the Cartagena Protocol on Biosafety requires parties to comply with information requirements set in Annex II, according to which states have provided information facility in the protocol, the biosafety clearing house, a risk assessment report according to guidelines established in AnnexIII.

Article 17 stated that the unintentional transboundary movements and emergency measures of the living modified organisms that likely have significant adverse effect on the conservation and sustainable use of biological diversity take into account risk to human health.

Article 18 of the Caratagena Protocol addressing handling transport, packaging, and identification of transboundary movements of LMOs for intentional introduction into the environment must identify the organisms as LMOs. The objective of this article is to make sure that the LMOs are handled and moved safely to avoid adverse effects on biological biodiversity and human health.

The precautionary principle of the Cartagena Protocol of Biosafety in Articles 5 and 6 mainly contained the advance 
information agreement and the risk assessment requirement. The inclusion of precautionary principle in the agreement to some academics contains its strongest enunciation and has been regarded "operationalization" in the body of environmental treaty. The catalyst for the application of precautionary measures in this agreement is the risk assessment. If the risk assessment shows unacceptable level of risk, then the GMO in question could be opposed to introduce in the environment.

Thus, the precautionary principle is not panacea, and it will not change world overnight, but it can make a difference in the protection of human health and environment by providing the guidance to policy makers when considering threats posed by GMOs [70, 71].

\section{Conclusion}

The use of genetically modified organisms is important in order to meet increasing demands and improve existing conditions prevalent in our environment. We are at an anxious juncture where, on one hand, we are faced with unprecedented threats to human health and environment, while on the other hand we have opportunities to change the way things are done. Regulations concerning use of GMOs need a broader basis for decision. Postrelease impacts of GMOs can follow preventive and precautionary measures based on risk assessment and management. Monitoring and detection methods are vital for risk assessment and management to control the negative environmental and health impacts. The international biosafety regulatory frameworks are sufficiently stringent in order to protect against genuine ascertainable risks, as well as the ability of decision makers to discern the appropriateness of data necessary to adequately conduct a risk assessment, which all have considerable consequences. Consideration of social, economic, and ethical issues needs to be taken care of. Application of the precautionary approach provides avenues for future development and use of genetic engineering.

\section{Future Prospective of GMO}

Regulation of GMO deals with a transscientific problem, that is, the resolution of the problems is beyond the competence of the scientific system. Public perception and acceptance are dependent on trust and whether the products or processes benefit them as citizens and consumers. To take proper accounts of uncertainties and public concern would help to capture the benefits, minimize the risk, and provide goals for future development and use of genetic engineering. Judgment about risks should not be based on the method modification (classical or modern) but on the quality of the final product. What does the GMO contain, is it safe, and not how was the GMO made? Encouragement of new monitoring and detection methods and tools is therefore vital for assessment, control of environmental, and health impacts as well as collection of ecological knowledge of relevance to future releases.

\section{Acknowledgment}

Dhan Prakash, Ranjana Bhatia, and Sonika Verma contributed equally for the this review article.

\section{References}

[1] C. James, "Preview: global status of commercialized Biotech/ GM crops," ISAAA Brief no. 35, ISAAA, Ithaca, NY, USA, 2006.

[2] L. Alberghina, L. Frontali, and P. Sensi, Proceedings of the 6th European Congress on Biotechnology, Elsevier Science Publishers, Amsterdam, The Netherlands, 1994.

[3] R. A. Hill, "Conceptualizing risk assessment methodology for genetically modified organisms," Environmental Biosafety Research, vol. 4, no. 2, pp. 67-70, 2005.

[4] H. A. Kuiper and H. V. Davies, "The safe foods risk analysis framework suitable for GMOs? A case study," Food Control, vol. 21, no. 12, pp. 1662-1676, 2010.

[5] K. Birkenhead, Y. P. Wang, B. Noonan, S. S. Manian, and F. O'Gara, "Characterization of Rhizobium meliloti dct genes and relationships between utilization of dicarboxylic acid and expression of nitrogen fixation genes," in Advances in Molecular Genetics of Plant-Microbe Interactions, R. Verma and D. P. Palacios, Eds., p. 834, American Phytopathology Society, St Paul Publishers, 1990.

[6] A. H. Bosworth, M. K. Williams, K. A. Albrecht et al., "Alfalfa yield response to inoculation with recombinant strains of $R h i$ zobium meliloti with an extra copy of dctABD and/or modified nifA expression," Applied and Environmental Microbiology, vol. 60, no. 10, pp. 3815-3832, 1994.

[7] V. Corich, F. Bosco, A. Giacomini, M. Basaglia, A. Squartini, and M. P. Nuti, "Fate of genetically modified Rhizobium leguminosarum biovar viciae during long-term storage of commercial inoculants," Journal of Applied Bacteriology, vol. 81, no. 3, pp. 319-328, 1996.

[8] D. Schubert, "A different perspective on GM food," Nature Biotechnology, vol. 20, no. 10, p. 969, 2002.

[9] H. Heuer and K. Smalla, "Horizontal gene transfer between bacteria," Environmental Biosafety Research, vol. 6, no. 1-2, pp. 3-13, 2007.

[10] J. M. Tiedje, R. K. Colwell, and Y. L. Grossman, “The planned introduction of genetically engineered organisms: ecological considerations and recommendations," Ecology, vol. 70, no. 2, pp. 298-315, 1989.

[11] G. T. Tzotzos, G. P. Head, and R. Hull, "Principles of risk assessment," Genetically Modified Plants, vol. 2009, pp. 33-63, 2010.

[12] C. N. Stewart Jr., H. A. Richards, and M. D. Halfhill, "Transgenic plants and biosafety: science, misconceptions and public perceptions," BioTechniques, vol. 29, no. 4, pp. 832-843, 2000.

[13] European Food Safety Authority, "Guidance document of the scientific panel on genetically modified organisms for the risk assessment of genetically modified microorganisms and their derived products intended for food and feed use," EFSA Journal, vol. 374, pp. 1-115, 2006.

[14] K. L. Johnson, A. F. Raybould, M. D. Hudson, and G. M. Poppy, "How does scientific risk assessment of GM crops fit within the wider risk analysis?" Trends in Plant Science, vol. 12, no. 1, pp. 1-5, 2007.

[15] N. C. Ellstrand, H. C. Prentice, and J. F. Hancock, "Gene flow and introgression from domesticated plants into their wild 
relatives," Annual Review of Ecology and Systematics, vol. 30, pp. 539-563, 1999.

[16] C. Dennis, “The brave new world of RNA," Nature, vol. 418, no. 6894, pp. 122-124, 2002.

[17] W. Martin, "Mosaic bacterial chromosomes: a challenge en route to a tree of genomes," BioEssays, vol. 21, no. 2, pp. 99104, 1999.

[18] H. Ochman, J. G. Lawrence, and E. A. Grolsman, "Lateral gene transfer and the nature of bacterial innovation," Nature, vol. 405, no. 6784, pp. 299-304, 2000.

[19] P. M. Bennett, C. T. Livesey, D. Nathwani, D. S. Reeves, J. R. Saunders, and R. Wise, "An assessment of the risks associated with the use of antibiotic resistance genes in genetically modified plants: report of the Working Party of the British Society for Antimicrobial Chemotherapy," Journal of Antimicrobial Chemotherapy, vol. 53, no. 3, pp. 418-431, 2004.

[20] S. Yoshida, S. Maruyama, H. Nozaki, and K. Shirasu, "Horizontal gene transfer by the parasitic plant Striga hermonthica," Science, vol. 328, no. 5982, p. 1128, 2010.

[21] P. Keese, "Risks from GMOs due to horizontal gene transfer," Environmental Biosafety Research, vol. 7, no. 3, pp. 123-149, 2008.

[22] V. E. Prescott, P. M. Campbell, A. Moore et al., "Transgenic expression of bean $\alpha$-amylase inhibitor in peas results in altered structure and immunogenicity," Journal of Agricultural and Food Chemistry, vol. 53, no. 23, pp. 9023-9030, 2005.

[23] K. M. Nielsen and J. P. Townsend, "Monitoring and modeling horizontal gene transfer," Nature Biotechnology, vol. 22, no. 9, pp. 1110-1114, 2004.

[24] Gene Technology Ethics Committee, "Working paper: ethical issues arising from trans-species gene transfer," 2006, http://www.ogtr.gov.au.

[25] European Food Safety Authority, "Statement of the scientific panel on GMO on the safe use of the nptII antibiotic resistance marker gene in genetically modified plants," 2007, http://www.efsa.europa.eu/etc/medialib.

[26] M. Labra, C. Savini, M. Bracale et al., "Genomic changes in transgenic rice (Oryza sativa L.) plants produced by infecting calli with Agrobacterium tumefaciens," Plant Cell Reports, vol. 20, no. 4, pp. 325-330, 2001.

[27] W. Craig, M. Tepfer, G. Degrassi, and D. Ripandelli, "An overview of general features of risk assessments of genetically modified crops," International Journal of Biological Sciences, vol. 5, pp. 706-726, 2008.

[28] M. J. Wilkinson and C. S. Ford, "Estimating the potential for ecological harm from gene flow to crop wild relatives," Collection of Biosafety Reviews, vol. 3, pp. 42-47, 2007.

[29] K. S. Oberhauser, M. D. Prysby, H. R. Mattila et al., "Temporal and spatial overlap between monarch larvae and corn pollen," Proceedings of the National Academy of Sciences of the United States of America, vol. 98, no. 21, pp. 11913-11918, 2001.

[30] J. M. Pleasants, R. L. Hellmich, G. P. Dively et al., "Corn pollen deposition on milkweeds in and near cornfields," Proceedings of the National Academy of Sciences of the United States of America, vol. 98, no. 21, pp. 11919-11924, 2001.

[31] G. P. Dively, R. Rose, M. K. Sears et al., "Effects on monarch butterfly larvae (Lepidoptera: Danaidae) after continuous exposure to Cry1Ab-expressing corn during anthesis," Environmental Entomology, vol. 33, no. 4, pp. 1116-1125, 2004.

[32] J. D. Pidgeon, M. J. May, J. N. Perry, and G. M. Poppy, "Mitigation of indirect environmental effects of GM crops," Proceedings of the Royal Society B: Biological Sciences, vol. 274, no. 1617, pp. 1475-1479, 2007.
[33] J. E. Losey, L. S. Rayor, and M. E. Carter, "Transgenic pollen harms monarch larvae," Nature, vol. 399, no. 6733, p. 214 , 1999.

[34] J. D. Wolt, R. K. Peterson, P. Bystrak, and T. Meade, "A screening level approach for non-target insect risk assessment: transgenic Bt corn pollen and the monarch butterfly (Lepidoptera: Danaiidae)," Environmental Entomology, vol. 32, pp. 237-247, 2003.

[35] J. Morris, "The relationship between risk analysis and the precautionary principle," Toxicology, vol. 181-182, pp. 127130, 2002.

[36] M. P. Nuti, A. Squartini, and A. Giacomini, "European community regulation for the use and release of genetically modified organisms (GMOs) in the environment," in Molecular Ecology of Rhizosphere Microorganisms, F. O'Gara, D. N. Dowling, and B. Boesten, Eds., pp. 165-173, VCH Publishers, New York, NY, USA, 1994.

[37] M. R. Smith and A. König, "Environmental risk assessment for food-related substances," Food Control, vol. 21, no. 12, pp. 1588-1600, 2010.

[38] Y. Devos, P. Maeseele, D. Reheul, L. Speybroeck, and D. Waele, "Ethics in the societal debate on genetically modified organisms: a (Re)quest for sense and sensibility," Journal of Agricultural and Environmental Ethics, vol. 21, no. 1, pp. 2961, 2008.

[39] European Food Safety Authority, "Guidance document of the Scientific Panel on Genetically Modified Organisms for the risk assessment of genetically modified plants and derived food and feed," 2004, http://www.efsa.europa.eu/ en/science/gmo/gmo_guidance.html.

[40] D. A. Andow and C. Zwahlen, "Assessing environmental risks of transgenic plants," Ecology Letters, vol. 9, no. 2, pp. 196-214, 2006.

[41] Organization of Economic Corporation and Development, "Descriptions of selected key generic terms used in chemical hazard/risk assessment, OECD series on testing and assessment number 44, ENV/JM/ MONO(2003)15, environment directorate," in Proceedings of the Joint Meeting of the Chemicals Committee and the Working Party on Chemicals, Pesticides and Biotechnology, France, 2003.

[42] G. L. Lövei and S. Arpaia, "The impact of transgenic plants on natural enemies: a critical review of laboratory studies," Entomologia Experimentalis et Applicata, vol. 114, no. 1, pp. 1-14, 2005.

[43] S. Hassan, "The initiative of the IOBC/WPRS working group on pesticides and beneficial organism," in Ecotoxicology: Pesticides and Beneficial Organisms, P. Haskell and P. McEwan, Eds., pp. 22-27, Kluwer Academic Publishers, Dordrecht, Germany, 1998.

[44] M. Morgan and M. Henrion, Uncertainty: A Guide to Dealing with Uncertainty in Quantitative Risk and Policy Analysis, Cambridge University Press, Cambridge, UK, 1990.

[45] ACRE, "Managing the footprint of agriculture: towards a comparative assessment of risks and benefits for novel agricultural systems," in Proceedings of the Advisory Committee on Releases to the Environment, London, UK, 2006.

[46] G. M. Poppy, "Geneflow from GM plants—-towards a more quantitative risk assessment," Trends in Biotechnology, vol. 22, no. 9, pp. 436-438, 2004.

[47] United Nations Environment Programme, "Governance for sustainable and human development," UNDP policy document, 1997, http://mirror.undp.org/magnet/policy/chapter1 .htm. 
[48] K. R. Hayes, P. C. Gregg, V. V. Gupta et al., "Identifying hazards in complex ecological systems. Part 3: Hierarchical Holographic Model for herbicide tolerant oilseed rape," Environmental Biosafety Research, vol. 3, no. 2, pp. 109-128, 2004.

[49] World Trade Organization, "European communities—-measures affecting the approval and marketing of biotech products, panel report, WT/DS291/R, WT/DS292/R and WT/DS293/R final report," 2006, http://www.wto.org.

[50] C. N. Stewart Jr., "Gene flow and the risk of transgene spread," 2008, http://agribiotech.info/details/StewartGeneFlow\%20Mar\%208\%20-\%2003.pdf.

[51] R. I. Rose, "Tier-based testing for the effects of proteinaceous insecticidal plant-incorporated protectants on nontarget arthropods in the context of regulatory risk assessments," IOBC WPRS Bull, vol. 29, pp. 143-150, 2006.

[52] L. A. Kogan, "World Trade Organization biotech decision clarifies central role of science in evaluating health and environmental Risks for regulation purposes," Global Trade and Customs Journal, vol. 2, p. 149, 2007.

[53] J. D. Wolt and R. K. D. Peterson, "Agricultural biotechnology and societal decision-making: the role of risk analysis," AgBioForum, vol. 3, no. 1, pp. 291-298, 2000.

[54] A. I. Myhr and T. Traavik, "The precautionary principle: scientific uncertainty and omitted research in the context of GMO use and release," Journal of Agricultural and Environmental Ethics, vol. 15, no. 1, pp. 73-86, 2002.

[55] O. Renn, "Precaution and ecological risk," in Encyclopedia of Ecology, vol. 4, pp. 2909-2916, 2008.

[56] M. Garcia-Alonso, E. Jacobs, A. Raybould et al., "A tiered system for assessing the risk of genetically modified plants to non-target organisms," Environmental Biosafety Research, vol. 5, no. 2, pp. 57-65, 2006.

[57] S. Mayer and A. Stirling, "Finding a precautionary approach to technological developments-lessons for the evaluation of GM crops," Journal of Agricultural and Environmental Ethics, vol. 15 , no. 1 , pp. 57-71, 2002.

[58] J. K. Jansson, "Tracking genetically engineered microorganisms in nature," Current Opinion in Biotechnology, vol. 6, no. 3, pp. 275-283, 1995.

[59] R. Costanza, "Visions, values, valuation, and the need for an ecological economics," BioScience, vol. 51, no. 6, pp. 459-468, 2001.

[60] United Nations Environment Programme, "Rio declaration on environment and development," in Proceedings of the United Nations Conference on Environment and Development, Rio de Janeiro, Brazil, June 1992.

[61] E. Fisher and R. Harding, Perspectives on the precautionary principle, Federation Press, Sydney, Australia, 1999.

[62] C. Raffensberger and J. Tickner, Protecting Public Health and the Environment: Implementing the Precautionary Principle, Island Press, Washington, DC, USA, 1999.

[63] G. Conko, "Safety, risk and the precautionary principle: rethinking precautionary approaches to the regulation of transgenic plants," Transgenic Research, vol. 12, no. 6, pp. 639647, 2003.

[64] I. M. Goklany, The improving state of the world: why we're living longer, healthier, more comfortable lives on a cleaner planet, Cato Institute, Washington, DC, USA, 2007.

[65] M. Marvier, C. McCreedy, J. Regetz, and P. Kareiva, "A metaanalysis of effects of Bt cotton and maize on nontarget invertebrates," Science, vol. 316, no. 5830, pp. 1475-1477, 2007.
[66] B. Durodie, Poisonous Dummies: European Risk Regulation after BSE, European Science and Environment Forum, Cambridge, UK, 1999.

[67] J. Tait, "More faust than frankenstein," Journal of Risk Research, vol. 4, pp. 175-191, 2001.

[68] Commission of the European Communities, "Communication from the commission on the precautionary principle," Tech. Rep. 2.2.2000 COM, European Comission, Brussels, Belgium, 2009.

[69] "Wingspread conference on the precautionary principle," 1998, http://www.sehn.org/wing.html.

[70] J. N. Herrera, "International law and GMOs: can the precautionary principle protect the biological diversity," Boletin Maxicano de Derecheo Comparado, vol. 118, pp. 97-136, 2007.

[71] T. E. Nickson, "Planning environmental risk assessment for genetically modified crops: problem formulation for stresstolerant crops," Plant Physiology, vol. 147, no. 2, pp. 494-502, 2008 . 

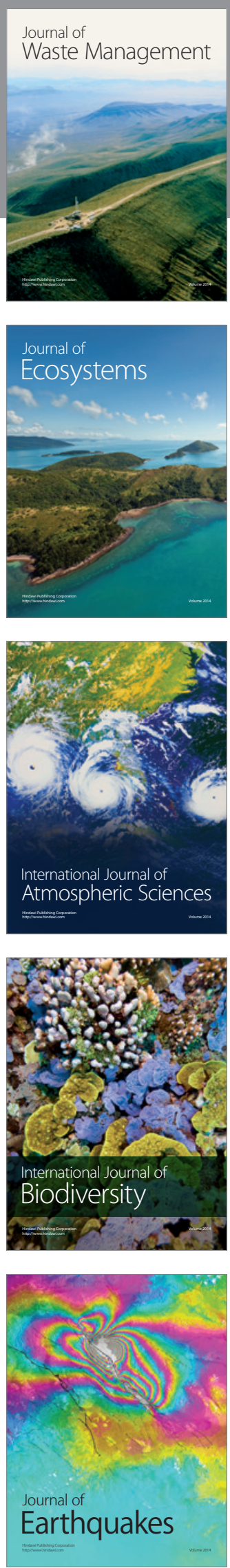
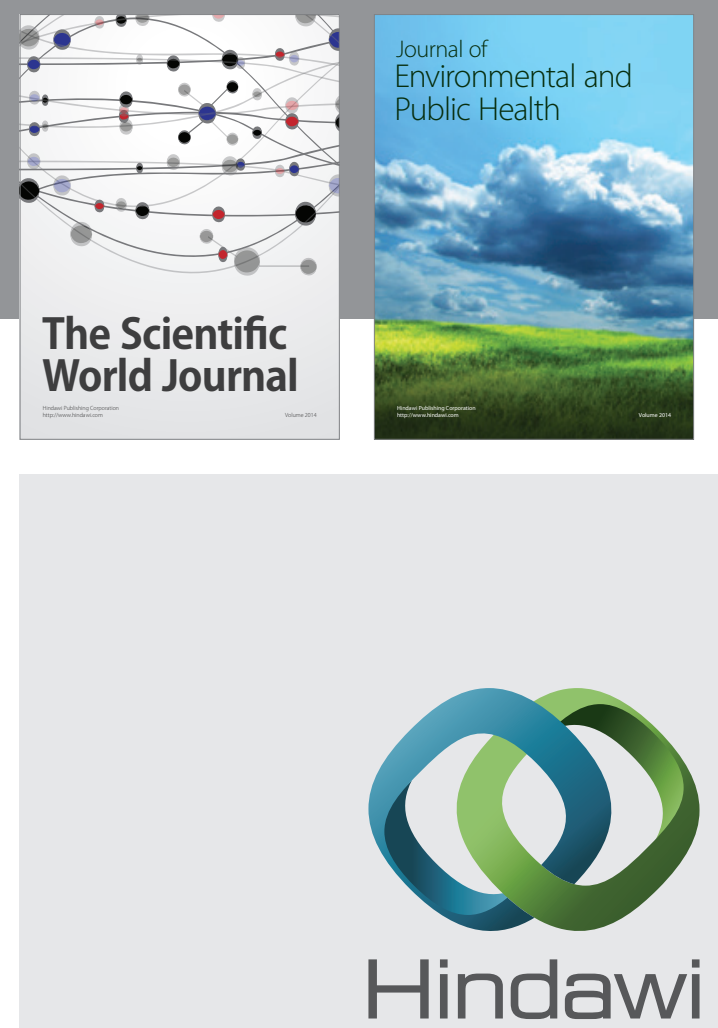

Submit your manuscripts at

http://www.hindawi.com
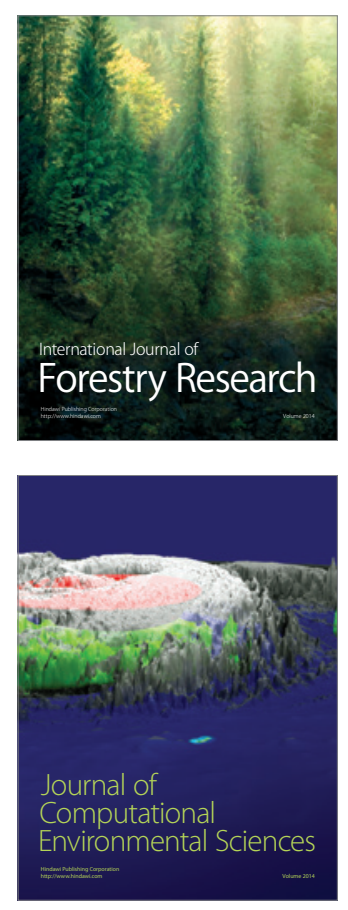
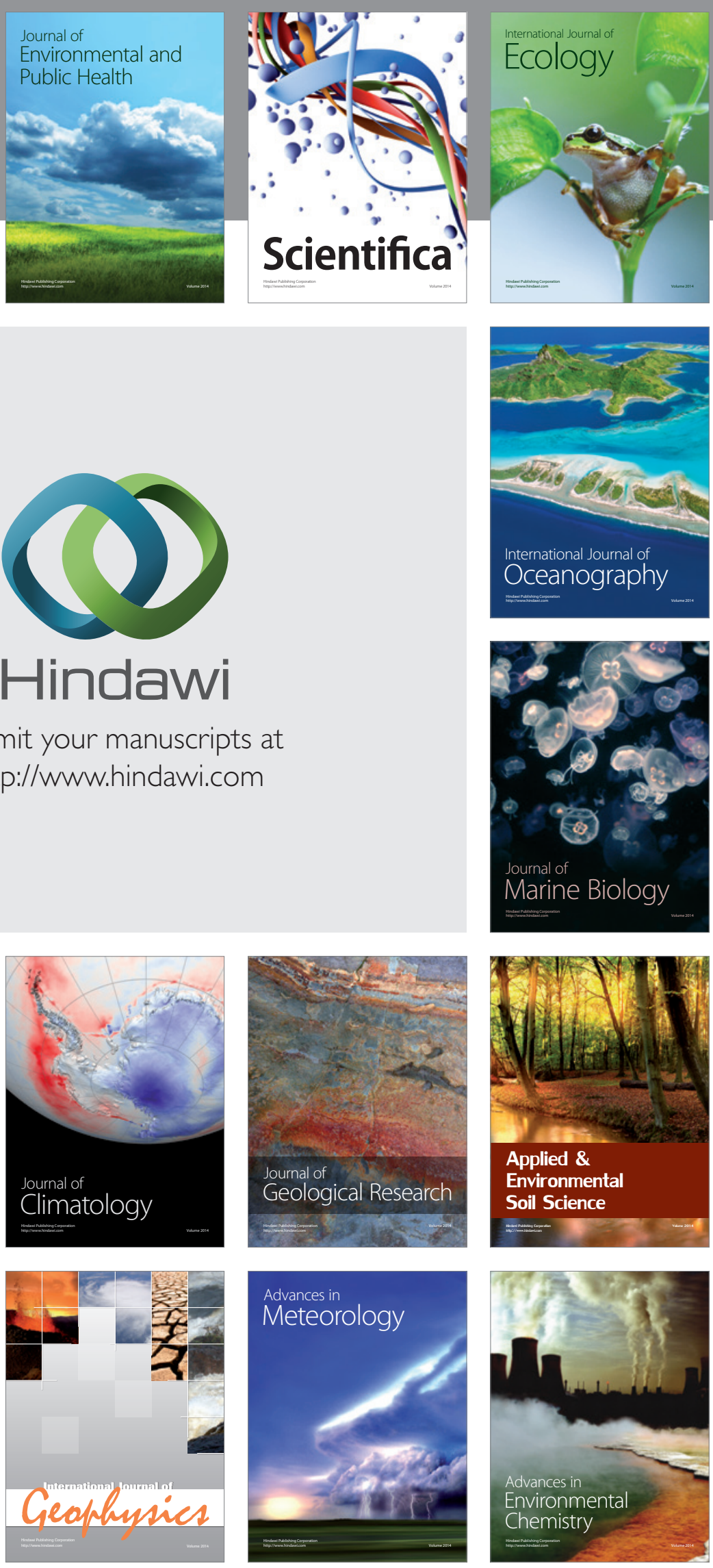